\title{
RNA interference: What and why?
}

\author{
Deepu Pandita* \\ Government Department of School Education, Jammu and Kashmir, India
}

Accepted on January 09, 2018

\section{Editorial}

RNA interference is a highly evolutionarily conserved cellular mechanism of gene regulation in which the own sequences of a gene turn it off. Also, the exogenous double-stranded RNA can suppress the expression of a particular gene which may correspond to that. It is also known as RNAi, RNA silencing, gene silencing, posttranscriptional gene silencing or quelling. By it the unwanted or harmful genes get turned/ switched off or silenced. Napoli and Jorgensen in order to intensify the color of petunias [1] inserted a gene for color in it, which to their surprise turned off the gene. Several years after the Petunia experiments it was revealed that the double-stranded RNA (dsRNA) turn off genes in a cell which is threatened or damaged by invading viruses. RNAi was first discovered by Andrew Fire and Craig Mello in Caenorhabditis elegans [2] for which they received Noble Prize in Physiology or Medicine [3]. This mechanism is today known in a wide variety of prokaryotic and eukaryotic organisms including mammals.

RNAi occurs at the post-transcriptional level and is triggered by short double-stranded RNA (dsRNA) 21 nucleotides long also known as short/small interfering RNA (siRNA) which is diced/ cut from long dsRNA by the RNase III enzyme Dicer or can also be introduced into a cell exogenously. Then siRNA binds to an RNA-Induced Silencing Complex (RISC) which separates the two strands into the passenger and guide strand. The passenger strand is degraded/removed while the RISC takes the remaining guide strand to a specific mRNA target site, cleaving it so that the unwanted target protein is not produced. This is how the gene is 'silenced Once bound, the Argonaute protein can either cleave the messenger RNA leading to sequence-specific degradation of its homologous mRNA sequences, destroying it, or recruit accessory factors to regulate the target sequence in other ways of siRNA pathway, miRNA Pathway and piRNA pathway.

RNAi is a sophisticated cellular mechanism for regulating a number of biological processes like defense against transposable elements and viruses, chromosome structure and stability maintenance and developmental timing and differentiation regulation. The genome of organisms has genes for miRNAs which are innate form of developmentally-important siRNAs. The cellular processing of miRNAs differs from siRNAs in the way that these are derived from single-stranded RNAs that fold back on themselves to generate small "stem-loops" of RNA. MicroRNAs when in RISC complex repress messenger RNAs which are incompletely complimentary to it that is why single miRNAs can regulate hundreds of genes. Human beings produce $\sim 500$ miRNAs and the inapt formation of specific miRNAs cause many human diseases.

\section{Biological Functions}

\section{Immunity}

RNAi is an adaptive immune system mechanism which evades viruses, exogenous genetic material and transposon proliferation in various organisms [4-9].

\section{Gene regulation}

Both the intronic and intergenic miRNAs on endogenous expression lead to translational repression or down regulation of genes [10]. The siRNA and miRNA when complementary to parts of a promoter can cause RNA activation or up regulation of genes leading to enhancement in gene transcription by histone demethylation [11] or by some unknown mechanism [12,13].

\section{Heterochromatin formation}

Two important properties that define heterochromatin involve its modes of assembly and inheritance. Heterochromatin is formed at repetitive DNA sequences along with transposons with function in chromosome transmission, genomic stability maintenance and gene expression regulation. The RNAinduced transcriptional silencing (RITS) complex which is Ago1-containing effector is guided to precise chromosomal locations of centromeric repeats by its bound siRNAs. siRNA recognize nascent transcripts by direct interaction between RITS and RNA polymerase II. RITS association promotes histone $\mathrm{H} 3$ methylation on lysine 9 (H3K9) which leads to the conscription of chromo domain-containing protein Swi6 and successive chromatin condensation. RITS moreover activates the RNA-dependent RNA polymerase complex (RDRC) which employs RdRP subunit (Rdp1) to produce secondary siRNAs for silencing. This RNAi positive feedback loop then employs the CLRC H3K9 methyl-transferase, two HP1 proteins (Swi6 and Chp2) which endorse the association of RITS with the noncoding RNA or mediate TGS by conscription of the SHREC2 deacetylase complex respectively. TRAMP/exosome pathway is another factor for the degradation of heterochromatic transcribes, which further ensure gene silencing [5].

\section{Applications}

\section{Gene knockdown}

Scientific researchers employ the RNAi pathway in vitro to silence genes for knowing their function. The dsRNA may be synthetic (siRNA) or single stranded RNA (ssRNA) and or expressed from a DNA construct which pierces the cytoplasm. Today commercially synthesized siRNAs are accessible to silence virtually any gene in any organism which has radically accelerated the speed of biomedical research. Various infectious 
diseases or genetic disorders such as many cancers and neurodegenerative diseases which result from the inappropriate and undesirable activity of a gene find solution in RNAi which is appearing as a modern therapeutic approach because of its ability to turn off expression of a single gene. Several clinical trials are going on for scrutiny of the safety and effectiveness of siRNA drugs [14].

\section{Functional genomics}

C. elegans and Drosophila melanogaster are most commonly used animal models for studying the functional genomics applications of RNAi $[15,16]$, while in hexaploid bread wheat [17] and other plant model systems like Arabidopsis thaliana and Zea mays [18] RNAi is successful. The cotton stocks have been produced with the application of RNAi which have reduced levels of delta-cadinene synthase, a key enzyme for gossypol production in seeds [19]. Other plants employing the use of RNAi are cassava plants without cyanogenic natural product linamarin [20], tomato plants with reduced levels of allergens [21] and fortified tomatoes with dietary antioxidants [22]. Today Flavr Savr tomato and ring spot-resistant papaya cultivars are already obtainable commercial crops [23,24].

\section{Loss of function studies}

siRNA libraries are used to study gene function in number of process like viability, fat metabolism, and embryogenesis. RNAi is useful for researchers to study reverse genetics and for identifying new molecules involved in cell death regulation and proliferation.

\section{Medicine}

The introduction of short interfering RNA into mammalian cells [25] is applicable. There are several disease-causing microRNAs causing human diseases which are now being tested as target therapies by drugs to deactivate them. RNAi has antiviral and anticancer applications and reverses induced liver failure in mouse models [26], [27]. The therapeutic treatment of macular degeneration and respiratory syncytial virus via RNAi are under clinical trials [28].

Cancer treatment: RNAi can be exploited for therapy of leukemia and lymphomas by targeting Bcr/Abl, NPM/ALK and other oncogenic fusion transcripts- with over expression in cancerous cell. siRNA-mediated gene silencing is highly sequence specific, meaning that only a perfect match between the siRNA and its target leads to silencing. Consequently, synthetic siRNA can be designed to silence cancer-derived transcripts which will be highly selective. Only cancer cell transcribes the mutated version of the gene, dsRNA is generated in only these cells. Therefore, only in these cells PKR is activated.

AIDS treatment: Human Immunodeficiency Virus (HIV) was the first infectious agent targeted by RNAi, as the lifecycle and gene expression pattern of HIV is identified. Synthetic siRNAs have been used to target several early and late HIV-encoded RNAs in cell lines and in primary hematopoietic cells which include the TAR element 50, tat51-53, rev51,52, gag54,55, env55, vif50, nef50, and reverse transcriptase 53 NF-KB, macrophage co receptor CCR5 [29].
In short, the biological role of silencing and its applications are:

- Protection against invasive RNAs especially RNA viruses

- Silencing of transposable elements

- Regulation of expression of endogenous genes

- Heterochromatin modeling

- Functional analysis of genes

- Sequence specific suppression of unwanted genes

- Generation of plants resistant to viruses

- A useful approach in future gene therapy

\section{References}

1. Napoli C, Lemieux C, Jorgensen R. Introduction of a chimeric chalcone synthase gene into petunia results in reversible co-suppression of homologous genes in trans. Plant Cell. 1990;2:279-89.

2. Fire $\mathrm{A}, \mathrm{Xu} \mathrm{S}$, Montgomery $\mathrm{MK}$, et al. Potent and specific genetic interference by double-stranded RNA in Caenorhabditiselegans. Nature. 1998;391:806-11.

3. Cejka D, Losert D, Wacheck V. Short interfering RNA (siRNA): Tool or therapeutic? Clinical Sci. 2006;110:4758.

4. Blevins T, Rajeswaran R, Shivaprasad P, et al. Four plant dicers mediate viral small RNA biogenesis and DNA virus induced silencing. Nucleic Acids Res. 2006;34:6233-46.

5. Voinnet O. RNA silencing as a plant immune system against viruses. Trends Genet. 2001;17:449-59.

6. Zambon R, Vakharia V, Wu L. RNAi is an antiviral immune response against a dsRNA virus in Drosophila melanogaster. Cell Microbiol. 2006;8:880-9.

7. Wang X, Aliyari R, Li W, et al. RNA interference directs innate immunity against viruses in adult drosophila science. 2006;312:452-4.

8. Lu R, Maduro M, Li F, et al. Animal virus replication and RNAi mediated antiviral silencing in $C$ elegans. Nature. 2005;436:1040-3.

9. Wilkins C, Dishongh R, Moore S, et al. RNA interference is an antiviral defense mechanism in Caenorhabditiselegans. Nature. 2005;436:1044-7.

10. Saumet A, Lecellier CH. Anti-viral RNA silencing: Do we look like plants? Retrovirology. 2006;3:3.

11. Check E. RNA interference: hitting the on switch. Nature. 2007;448:855-8.

12. Li LC, Okino ST, Zhao H, et al. Small dsRNAs induce transcriptional activation in human cells. Proc Natl Acad Sci U.S.A. 2006;103:17337-42.

13. Vasudevan S, Tong Y, Steitz JA. Switching from repression to activation: MicroRNAs can up- regulate translation. Science. 2007;318:1931-4. 
14. Daneholt B. Advanced Information: RNA interference. The Nobel Prize in Physiology or Medicine; 2006.

15. Kamath R, Ahringer J. Genome-wide RNAi screening in Caenorhabditis elegans. Methods. 2006;30:313-21.

16. Boutros M, Kiger A, Armknecht S, et al. Genome-wide RNAi analysis of growth and viability in Drosophila cells. Science. 2004;303:832-5.

17. Travella S, Klimm T, Keller B. RNA Interference-based gene silencing as an efficient tool for functional genomics in hexaploid bread wheat. Plant Physiol. 2006;142:6-20.

18. McGinnis $\mathrm{K}$, Chandler V, Cone $\mathrm{K}$, et al. Transgene induced RNA interference as a tool for plant functional genomics. Methods Enzymol. 2005;392;1-24.

19. Sunilkumar G, Campbell L, Puckhaber L, et al. Engineering cotton seed for use in human nutrition by tissue-specific reduction of toxic gossypol. Proc Natl Acad Sci USA. 2006;103:18054-9.

20. Siritunga D, Sayre R. Generation of cyanogens free transgenic cassava. Planta. 2003;217:367-73.

21. Le L, Lorenz Y, Scheurer S, et al. Design of tomato fruits with reduced allergenicity by dsRNAi-mediated inhibition of ns-LTP (Lyce 3) expression. Plant Biotechnol J. 2006;4:231-42.
22. Niggeweg R, Michael A, Martin C. Engineering plants with increased levels of the antioxidant chlorogenic acid. Nat Biotechnol. 2004;22:746-54.

23. Sanders R, Hiatt W. Tomato transgene structure and silencing. Nat Biotechnology. 2005;23:287-9.

24. Chiang C, Wang J, Jan F, et al. Comparative reactions of recombinant papaya ringspot viruses with chimeric coat protein $(\mathrm{CP})$ genes and wild type viruses on $\mathrm{CP}$-transgenic papaya. J Gen Virol. 2001;82:2827-36.

25. Paddison P, Caudy A, Hannon G. Stable suppression of gene expression by RNAi in mammalian cells. Proc Natl Acad Sci USA. 2002;99:1443-8.

26. Sah D. Therapeutic potential of RNA interference for neurological disorders. Life Sci. 2006;79:1773-80.

27. Zender L, Hutker S, Liedtke C, et al. Caspase 8 small interfering RNA prevents acute liver failure in mice. Proc Natl Acad Sci USA. 2003;100:7797-802.

28. Whitehead KA, Dahlman JE, Langer RS, et al. Silencing or stimulation? siRNA delivery and the immune system. Ann Rev Chem Biomol Engineering. 2011;2:77-96.

29. Kumar P, Ban HS, Kim SS, et al. T cell-specific siRNA delivery suppresses HIV-1 infection in humanized mice. Cell. 2008;134:577-86.

\section{*Correspondence to:}

Deepu Pandita

Government Department of School Education

Jammu and Kashmir

India

Tel: 0194-2450738

E-mail: deepupandita@gmail.com 